ISBN 82-553-0332-4

Applied Mathematics

No 4 - December ?

1977

THREE-DIMENSIONAL LEE-WAVE PATTERN

by

B. Gjevik and T. Marthinsen

Oslo

Oslo

PREPRINT SERIES - Matematisk institutt, Universitetet i Oslo 


\title{
THREE-DIMENSIONAL LEE-WAVE PATTERN
}

\author{
B. Gjevik and T. Marthinsen \\ Inst1tute of Mathematics \\ University of 0sio, Norway
}

\section{Abstract}

Satelifte photographs are analysed in order to study the leewave pattern generated by isolated islands in the Norwegian Sea and the Barents Sea.

In situations where thewat motion is confined to the lower atmosphere (trapped waves) the waves are located within a wedge-shape wake behind the islands. Both the diverging wave type where the crests are orientated outward from the centre of the wake and the transverse wave type where the crests are nearly perpendicular to the wind direction are observed. The former wave type is, however, the most common wave type. In certain situations a long single-crested wave is observed at Jan Mayen. The wave appears on the photographs as a straight lane in the cloud layer. In one case it extends sidewise from the Island to a distance of about $350 \mathrm{~km}$. Wave kinematics is used to obtain the phase lines for steady wave pattern for different atmospheric models. The theory is found to explain some important features of the observed wave forms. 


\section{Introduction}

A vast literature is available on atmospheric lee-waves. The most of It is concerned with waves generated by mountain ridges where the motion of an individual air particle is essentially confined to a vertical plane perpendicular to the ridge. The three-dimensional wave motion which occurs when the waves are generated by isolated mountain peaks, have recelved considerably less attention. A general mathemat1cal treatment of this problem is very involved. The few works we know of, are Kochin (1938), Scorer and Wilkinson (1956), Wurtele (1957), Palm (1958), Crapper (1962), and Blumen \& McGregor (1976). These works are mainly theoretical studies of the wave motion for rather special atmospheric situation, and the results are not compared w1th observations. The works by Kochin, Scorer and Crapper show that trapped waves are confined to a wedge-shape region behind the wave source and that the horizontal structure of the wave pattern is similar to the well-known shlp-wave pattern. The waves are of two types: transverse waveswith the wave crest nearly perpendicular to the wind direction, and diverging waves which radiate out from the wake. Scorer et.al. (1956) remark that the latter wave type is unlikely to be observed in the atmosphere.

We have studied satellite photographs of lee-waves generated by isolated islands in the Norwegian Sea and the Barents Sea and the observational results are presented in Section 2. Contrary to Scorer's statement the diverging wave type is found to be rather common.

Since the existing theoretical results refer to atmospheric models which are not applicable in our case, we have used wave kinematics to obtain the phase Iines of the waves for more realistic atmospheric models. (Section 3). We have found that the theoretical results which 
are based on I1nearized wave theory, explain many features of the observed wave pattern. There are, however, discrepancies between theory and observation and the theory is unable to explain the singlecrested wave which can be observed at Jan Mayen in certain situations. Th1s wave form has to our knowledge not previously been described in the Iiterature.

\section{Observational results}

Studies of the satellite photographs show that three isolated islands in the Norweglan Sea and The Barents Sea often generate atmospheric lee-waves. These islands are $\operatorname{Jan}$ Mayen $\left(71,0^{\circ} \mathrm{N}, 8.5^{\circ} \mathrm{W}\right)$, The Bear-Island $\left(74.4^{\circ} \mathrm{N}, 19.0^{\circ} \mathrm{E}\right)$ and Hopen $\left(76.6^{\circ} \mathrm{N}, 25.3^{\circ} \mathrm{E}\right)$. Waves are particularly frequent in the wake of Jan Mayen. Th1s 1sland is also known to generate vortex-streets and the vortex shedding at Jan Mayen has been studied by Wehner (1949), Mohr (1971) and Fjellhelm (1973).

Before we proceed we will give a short description of the topography of the three islands.

In the eastern part of Jan Mayen a volcanfc mountain, Beerenberg, rises to a height of $2277 \mathrm{~m}$. The mountain is nearly conlc with a diameter at the foot of about $15 \mathrm{~km}$. Also the western part of the 18 land is mountalnous with the highest top at $769 \mathrm{~m}$. The terrain between the mountains in the east and the west is low.

There is a low plateau at about $30-40 \mathrm{~m}$ in the north-western part of The Bear Island. The south-eastern part of the island is mountainous with three main peaks respectively at $536 \mathrm{~m}, 440 \mathrm{~m}$ and $360 \mathrm{~m}$. 
The island Hopen consists of a long narrow mountain ridge with mountain tops between 250 and $370 \mathrm{~m}$.

In Fig. 1 the main topographical features of the three islands are shown.

On all three islands the Norwegian Meteorological Institute operates meteorological stations. From Hopen only surface observations are avallable, but from The Bear Island and Jan Mayen one also has regular upper air soundings.

We have examined VHRR-photographs in the visual and infrared band, taken by the sateliftes NOAA 4 and NOAA 5 in the period August 1976 to September 1977. Unfortunately, the photographs ava1lable to us are geometrically distorted, and data read from the photographs are corrected for the scale distortion. We have found lee-waves at Jan Mayen on 7 days, at The Bear Island on 2 days and at Hopen on 1 day. Vortex shedding at Jan Mayen occurred on 11 days.

Here we choose to present four cases with particular well developed lee-wave pattern. We belleve that these cases demonstrate typ 1cal and general features of the wave motion and we w1ll glve an analysfs of these cases.

Case I Jan Mayen, Sept. 1, 1976, about 11.17 GMT.

A photograph of the Jan Mayen region with a remarkable lee-wave pattern is shown in Figure 2a. The waves are visualized by a cloud layer, and the waves are clearly generated by the 1sland's influence on the air flow. The snow-covered top of Beerenberg appears on the photograph as a white spot at the upstream front of the wave train.

Figure $3 a$ shows a display of upper air data recorded by a radiosonde at nearly the same time as the photograph. These observations 
show that a temperature inversion extends between a helght of about $1400 \mathrm{~m}$ to about $2600 \mathrm{~m}$ and that the wind direction in the lower part of the atmosphere is nearly constant.

The surface observator reports cumulus and cumulonimbus clouds with cloud base at $300-600 \mathrm{~m}$ helght. The radiosonding shows that the a1r above $1800 \mathrm{~m}$ is very dry, and that the cloud layer appearing on the photograph is formed by the horizontal spreading at the inversion.

A striking feature of the wave pattern is the lack of symmetry and this indicates that the terrain on the 1sland has an important effect on the wave pattern.

The line formed by the converging wave crests at the centre of the wake corresponds closely to the wind direction above the cloud layer. We will therefore refer to this line which extends southward from the top of Beerenberg as the centre-axis of the wake.

A close inspection of the photograph reveals that an abrupt change in the wave-phase takes place along a stralght line west of the centre-axis. This indicates that the wave pattern west of the centre-axis consists of two wave trains of different origin. The western wave train is most likely generated by the mountains on the western part of Jan Mayen while the wave train diverging out from the centre axis is generated by Beerenberg. The latter wave train is confined within a sector of about $17^{\circ}$. We measured the wave-length in the front of western wave train to about $9 \mathrm{~km}$ and found the wave crests to make an angle of about $30^{\circ}$ with the centre axis. Assuming stationary wave crests, this leads to a phase velocity of about $18 \mathrm{~m} / \mathrm{s}$.

Within a sector of about $24^{\circ}$ east of the centre axis and close to the island, there is a train of diverging waves. These waves are clearly generated by Beerenberg and they seem to be similar to the 
waves generated on the western side of the mountain.

A straight dark lane in the cloud layer radiates from the eastern slope of Beerenberg to a distance of about $350 \mathrm{~km}$ from the 1sland. The clearance in the cloud layer is most likely caused by downward motion of the air. The width of the lane is about $14 \mathrm{~km}$ and it makes an angle of about $52^{\circ}$ with the wind direction. If the lane is stationary with respect to the ground, the propagation velocity relative to the air flow is about $27 \mathrm{~m} / \mathrm{s}$. Linear wave theory (see section 4) is unable to explain this high propagation velocity and also the fact that the lane appears to propagate with a nearly unchanged form. We suggest that this single crested wave may have a similar origin and structure as the solitary wave studied by Davis \& Acrivos (1967). They found that a steady non-I1near wave can propagate along an interfacial transition region between two homogeneous layers and that the propagation velocity for this wave increases with increasing amplitude of the wave.

Case II Jan Mayen, Dec. 29, 1976, 11.22 GMP.

Also on this photograph (Fig. 2b) a straight clear lane in the cloud layer radiates out from the eastern side of Jan Mayen where the snow-covered top of Beerenberg can be seen as a weak white spot. The wave length (width of the lane) is found to be about $14 \mathrm{~km}$ and $1 t$ makes an angle of about $38^{\circ}$ with the wind direction. The propagation velocity (with respect to the air) is found under the assumption that the lane remains stationary with respect to the island to be about $11 \mathrm{~m} / \mathrm{s}$. We note that although the wave-length is the same in Case I and Case II, the propagation velocity is considerably lower in the latter case. 
The upper air data recorded at nearly the same time as the photograph is displayed in Fig. 3b. The wind direction varies little with height, and the wind is from the same direction as in Case I. As in Case I there is an low level temperature inversion, but in this case in only extend from $840 \mathrm{~m}$ to $1330 \mathrm{~m}$ above sea level.

The clouds over Jan Mayen are reported to be cumulus and cumulonimbus clouds, the cloud base is at $200-300 \mathrm{~m}$. The air above $40 \mathrm{~m}$ is very dry and it is unlikely that the top of the clouds penetrates the inversion layer. Due to heating of the air over the sea, cloud bands and cloud cells associated with convection currents in the air are seen on the photograph.

A high cloud (white on the photograph) is formed over the southwestern part of the 1sland. It is an indication of strong vertical motion over the mountains in this part of the island. The wavy cloud band downwind from Beerenberg indicates that the wake is unstable and the atmospheric conditions are close to those which lead to vortex shedding. In other situations where a shallow stable layer intersects Beerenberg in a similar way as in this case, vortex shedding is frequent, (Fjellhelm, 1973). The serles of satelitte photographs which we have examined, confirms this result. In none of these cases lee-waves are found to be exclted.

Case III Jan Mayen, Oct. $8,1976,13.14$ OMT.

In this case the wind is from east and there is an isothermal layer between $1720 \mathrm{~m}$ and $2600 \mathrm{~m}$ above sea level. (Fig. 3c). The cloud base (patches of stratus) is reported to be at 100-200 $\mathrm{m}$ with a layer of altocumulus clouds above. Above $1720 \mathrm{~m}$ the air is dry and we therefore assume that this level represents the top of the cloud layer. 
The photograph (Fig. 2c) shows that these clouds are formed at the crests of the waves, and that a wake 1s formed by higher clouds (white on the photograph). The wave crests on the southern side of the wake extend further outward from the wake than the wave crests on the northern side of the wake. This may be an effect of the mountaina on the southwestern part of Jan Mayen. A close inspection of the photograph also reveals a slight distortion of the wave crests. The waves on the northern side of the wake is confined within a sector of about $12^{\circ}$. If we disregard the distortion of the crests on the southern side of the wake, the wave pattern is remarkably symmetric on the two sides of the wake.

Case IV The Bear Island and Hopen, Sept. 19, 1976, 11.37 GMT.

The satalitte photograph from the spitebergen region (PIg. 2d) shows two lee-wave patterns southeast of Sp1tzbergen. The northern of these patterns is generated by Hopen and the southern pattern by The Bear Island. Upper air data from The Bear Island recorded at about the same time as the photograph are presented in FIg. 3d. A strong temperature inversion extends between a he1ght of about $700 \mathrm{~m}$ to a height of about $1500 \mathrm{~m}$. Fog is observed at the ground and the ar is saturated up to about $800 \mathrm{~m}$. Above this level the air is dry. The waves are therefore most likely visualized by a cloud layer at about $800 \mathrm{~m}$. The wind direction is from WNW and varies little with height. The wave pattern at The Bear Island consists of straight crested waves. The wave crests make an angle of about $65^{\circ}$ with the wind direction. The wave crest on the northern side of the centre of the wake extends further out than on the southern side, and the former waves are confined to a sector of about $21^{\circ}$. This asymmetry in the wave pattern may be due to the shape and the location of the three 
mountaln tops on The Bear Island.

The average wave length measured along the centre of the wake is about $8.5 \mathrm{~km}$.

The waves generated at Hopen is of the transverse wave type with wave crests almost perpendicular to the wind direction. The waves are confined within a sector of about $49^{\circ}$ and the average wave length along the centre-axis of the wave train is about $9.5 \mathrm{~km}$. There is no upper air data from Hopen. The temperature variation with helght may be nearly the same as at The Bear Island. Since Hopen is located leeward of Spitzbergen, the wind speed at Hopen is, however, most likely lower than at The Bear Island.

The four cases examined here show typical although remarkable well developed lee-wave pattern. We note that the waves generated at Jan Mayen are of the diverging wave type and that waves of the transverse wave type are only observed at Hopen. The wave pattern generated at The Bear Island is difficult to classify to either of these groups. Other photographs show that the topography of this island seems to favour wave pattern of this special form.

3 The lee-wave pattern from a point source. Wave kineatics.

Whitham (1974) surveys the theory and gives some illustrative examples on how wave pattern can be obtained by kinematic arguments. We will use the same technique to obtain the form of the lee-wave pattern for atmospheric models. We describe the motion from a fixed Cartesian coordinate system. The axes $x_{1}$ and $x_{2}$ are horizontal and the former axis is along the direction of the wind. The wave fleld is assumed to be steady and the wave parametres are almost periodic functions of the coordinates $x_{1}$ and $x_{2}$. We denote the phase 
function by $\psi\left(x_{1}\right) \quad(1=1,2)$ and define a local wave number vector by

$$
k_{1}=\frac{\partial \psi}{\partial x_{i}}
$$

The vector $k_{1}$ is therefore normal to curves of constant phase. The dispersion relation for IInear waves is a functional relation between $\mathbf{k}_{1}$ and the physical parametres defining the state of the atmosphere. We write the functional relation in the form

$$
D\left(k_{1}, U, N, H\right)=0
$$

where $U, N, H$ denote respectively the wind veloclty, the VäisallaBrunt frequency and the thickness of the layers in the atmosphere. (See Appendix). With the definition (3-1), equation (3-2) becomes a differential equation for $\psi$. This equation can be written in characterist1c form

$$
\frac{d x_{1}}{d \lambda}=\frac{\partial D}{\partial k_{i}} ; \quad \frac{d k_{i}}{d \lambda}=-\frac{\partial D}{\partial x_{i}} ; \quad \frac{d \psi}{d \lambda}=k_{j} \frac{\partial D}{\partial k_{j}}
$$

This set of equations determines the characteristics $x_{1}=x_{1}(\lambda)$, the phase function $\psi$, and the wave number vector $k_{1}$ in the hor1zontal plane. With horizontally unform layers it follows that $k_{1}$ is constant on the characteristics and for a point source the characteristics pass through this point. With the point source at origo, it follows from eq. $(3-3)$ that

$$
\frac{x_{2}}{x_{1}}=\left(\frac{\partial D}{\partial k_{2}} / \frac{\partial D}{\partial k_{1}}\right)
$$

and

$$
\psi=k_{i} x_{i}=2 n \pi \quad(n=0,1,2 \cdots)
$$

Eqs. $(3-4)-(3-5)$ determine the phase IInes. 
The discontinuous model leads to a relatively simple expression for $D$ (Appendix, eq. A1), and equations for the lines of constant phase can be obtained easily from eq. $(3-4)$ and $(3-5)$. We find

$$
\begin{aligned}
& \frac{x_{1}}{H}=p \frac{F^{2} \cos ^{3} \varphi\left(1-F^{2} \cos ^{2} \varphi+2 t g^{2} \varphi\right)}{\left(1-F^{2} \cos ^{2} \varphi\right)^{2}} \\
& \frac{x_{2}}{x_{1}}=-\frac{\operatorname{tg} \varphi\left(1+F^{2} \cos ^{2} \varphi\right)}{1-F^{2} \cos ^{2} \varphi+2 \operatorname{tg}{ }^{2} \varphi}
\end{aligned}
$$

where $p=2\left(\frac{U_{1}}{U_{0}}\right)^{2} n \pi$ and $-\frac{\pi}{2}<\varphi<\frac{\pi}{2} . F$ is the Froude number. In the limit $H \rightarrow \infty, F \rightarrow 0$ and eq. (3-6) reduces to the well-known equation for the phase IInes for ship-waves in deep water. For $F$ and $H$ finite the phase Iines obtained from eq. (3-6) are similar to the phase Iines for ship-waves in shallow water. For $F<1$ both transverse and diverging waves are possible. For $F>1$ there will only be diverging waves. The waves are confined within a wedge-shape region behind the wave source and the wedge-angle vary in a similar way as for ship-waves in shallow water. Subsequently we denote the semi wedge-angle by $\theta$. For $F=0,(H+\infty)=19.47^{\circ}$, for $F=1, \theta=90^{\circ}$ and for $F+\infty, \theta+0$.

Although the discontinuous model reveals characteristic features of the wave pattern, the model is too simple for a direct comparison with the observational result in section 2 . We have therefore investigated the properties of different four layer models. The dispersion relation for a four layer model is given in eq. A2 in the Appendix. This equation together with eqs. $(3-4)$ and $(3-5)$ determine the phase Ilnes which are evaluated by numerical methods.

In Figs. $4 \mathrm{a}$ and $4 \mathrm{~b}$ the computed values of the semi wedge-angle, $\theta$, as a function of the Scorer-parameter, $\gamma$, for the most stable layer are depicted. For these computations we have used layer thickness corresponding respectively to Case III and Case IV (See Table I). In F1gs. $4 \mathrm{a}$ and $4 \mathrm{~b}$ these models are marked by $\mathrm{a}$ dot. 
The curves label A (full Iine, 1.mode, dotted line, 2.mode) correspond to a model with $\gamma_{0}=\gamma_{2}=\gamma_{3}=0$, i.e. a three layer model where a stable layer is inbedded between two neutrally stable layers. For a certain value, $\gamma_{1}=\gamma_{1 \mathrm{~m}}$, say, the angle $\theta$ for the 1. mode approaches $90^{\circ}$. The diverging wave type is the only possible wave form for $\gamma_{1}<\gamma_{1 \mathrm{~m}}$. For $\gamma_{1}>\gamma_{1 \mathrm{~m}}$ both diverging and transverse waves are possible.

If the stability of the two upper layers are increased, the value of $\theta$ decreases. This effect is lllustrated by the curves labeled $B$ and $C$. The stable stratification of the upper layers allows long waves to propagate vertically and trapped waves are possible only for $r_{1}$ above a certain value and for wave-length shorter than a certaln cut-off. For simplicity reasons we denote $\gamma_{1} \cdot 10^{3} \mathrm{~m}$ by $\gamma_{1}$. In case B FIg. 4 a the diverging waves are the only possible wave form when $0.3<\bar{r}_{1}<0.6$. For $0.6<\bar{\gamma}_{1}<1.1$ both diverging and transverse waves are possible. The transverse waves disappear in a sector which includes the centre of the wake. For $\bar{r}_{1}>1.1$ there is no cut and both wave types are possible. Case $C$, Fig. (4a), is similar. For $0.5<\bar{r}_{1}<1.0$, diverging waves, for $1.0<\bar{\gamma}_{1}<1.2$, diverging and transverse waves, the latter waves disappear near the centre of the wake, and for $\bar{\gamma}_{1}>1.25$, diverging and transverse waves.

In the cases $B$ and $C, F 1 g$. (4b), the diverging and transverse waves are possible for $\bar{\gamma}_{1}>1.25$ and $\bar{\gamma}_{1}>1.45$ respectively. For $0.4<\bar{r}_{1}<1.2$, and $0.7<\bar{r}_{1}<1.4$, respectively, in case $B$ and $C$, both types is possible and the transverse waves are cut.

For the range of parametres treated in FIg. (4a) and (4b) the diverging wave type is the only possible wave form for the 2 . mode. 
The graphs corresponding to this mode are drawn by dotted lines.

For other four-layer models we have found quantitatively similar results as summarized in Figs. $4 \mathrm{a}$ and $4 \mathrm{~b}$.

$4 \quad$ Comparison between theory and observations

It should be stressed that the kinematic arguments give possible steady wave pattern due to a point source.

In order to determine uniquely which wave type is excited, the amplitudes of the waves have to be evaluated. This is a formidable problem which also requires a realistic representation of the topography of the wave source. The photographs of the wave pattern in Case I-IV indicate that at least in some of these situations the waves appear to be generated by a point source. We have therefore evaluated possible steady wave pattern for atmospheric models corresponding to Case I-IV by the methods of section 3 .

Since the wave motion is confined to the lower part of the atmosphere, we have disregarded the strong stability of the stratosphere. If this effect had been included in the models, the main effect would be a weak damping of the wave fleld in the horlzontal direction (slightly leaky waves).

The value of the parametres for the four models are given in Table I.

Table I

\begin{tabular}{|l|c|c|c|c|c|c|c|c|}
\hline Case & $\frac{\mathrm{U}}{\mathrm{U}_{0}}$ & $\begin{array}{c}\mathrm{H}_{0} \\
\mathrm{~km}\end{array}$ & $\begin{array}{c}\mathrm{H}_{1} \\
\mathrm{~km}\end{array}$ & $\begin{array}{c}\mathrm{H}_{2} \\
\mathrm{~km}\end{array}$ & $\begin{array}{c}\gamma_{0} 0^{10^{3}} \\
\mathrm{~m}^{-1}\end{array}$ & $\begin{array}{c}\gamma_{1} \cdot 10^{3} \\
\mathrm{~m}^{-1}\end{array}$ & $\begin{array}{c}\gamma_{2} \cdot 10^{3} \\
\mathrm{~m}^{-1}\end{array}$ & $\begin{array}{c}\gamma_{3}{ }^{-10^{3}} \\
\mathrm{~m}^{-1}\end{array}$ \\
\hline $\mathrm{I}$ & 1.1 & 0.9 & 1.7 & 2.0 & 0.2 & 0.5 & 0 & 0.2 \\
\hline II & 0.8 & 0.8 & 0.5 & - & 0.3 & 1.5 & 0.6 & - \\
\hline III & 0.8 & 1.7 & 0.9 & 6.7 & 0 & 0.9 & 0.4 & 0.5 \\
\hline IV & 1.0 & 0.7 & 0.8 & 1.7 & 0 & 1.5 & 0.3 & 0.55 \\
\hline
\end{tabular}


The phase-Iine pattern corresponding to these four models are displayed respectively in Fig. 5 a-d.

Case I The computed wave pattern consists mainly of diverging waves (F1g. 5a) and the semi wedge-angle is $17.8^{\circ}$. Also the photograph (Fig. 2a) shows a well developed train of diverging waves especially on the western side of the centre-axis of the wake. The part of the wave-train generated by Beerenberg seems to be confined within a wedge (See under Case $I$, section 2) and the wedge-angle and the length of the waves are in close agreement with the waves in Fig. 5a. The part of the wave-train generated by the mountains in the western part of the island are also confined within a wedge of similar width.

F1g. 5a shows that all the linear wave components of this model propagate relatively slow and the model is therefore unable to explain the existence of the single-crested wave east of Jan Mayen. (Fig. 2a). This leads us to suggest that the wave may be an internal solitary wave (See under Case I, section 2).

Case II The steady wave pattern (FIg. 5b) consists mainly of diverging waves. The semi wedge-angle is $15.3^{\circ}$ and the wave-length is shorter than $5 \mathrm{~km}$. The diverging wave type is not observed in this case nor in other similar situations (See under Case II, sectIon 2). These results indieate that wave components shorter than $5 \mathrm{~km}$, say, is unlikely to be exited when a relatively thin stable layer intersects Beerenberg well below the summit.

As in Case I the model is unable to explain the existence of the single-crested wave which can be seen east of Jan Mayen (Fig.2b). Case III In this case the steady pattern (Fig. 5c) consists only of diverging waves and the semi wedge-angle is $10.7^{\circ}$. This wave type 
1s also observed ( $F 1 g .2 c)$. The computed semi wedge-angle agrees well with the observed angle for the wave train north of the wake. The wave train south of the wake extends over a wider sector but this is most likely an effect of the mountains on the western part of the 1sland (See under Case III, section 2). We note that the observed wave length at the outer edge of the wedge compares well with the waves in Fig. 5c. The observed wave length near the centre of the wake is, however, somewhat larger than for the waves in Fig. $5 \mathrm{c}$.

Case IV In this case the computation leads to a wave pattern which consists both of diverging waves and well developed transverse waves (F1g. 5d). The seml wedge-angle 1s $21.7^{\circ}$ and the wave length of the transverse waves 18 about $10 \mathrm{~km}$. The wave length of the diverging waves is shorter. The transverse wave train compares relatively well with the observed wave train at Hopen. The straight-crested wave train generated at The Bear Island differs, however, from either of the wave trains in FIg. 5d. We note that the second mode for this model leads to straight crested waves, but other features of the wave train for this mode are in disagreement with the observation. The waves observed at The Bear Island may therefore be strongly effected by the topography of the island or eventually by a wind defect in its wake.

\section{Acknowledgement}

The satelilte photographs usea in this study are provided by Troms $\varnothing$ Sateli1tt- Telemetristasjon, NTNF, Troms $\phi$, Norway, and the meteorological data are provided by Norges Meteorologiske Institutt, Blindern, Norway.

We acknowledge the kindness and the expediency our many requests to these institutions have been met with. 


\section{APPENDIX}

We assume that the three-dimensional wave motion is steady, adlabatic and frictionless. The governing equation for this motion is given by the authors referred to in the introduction, and it is no need for recapitulating it here.

Since the wind velocity, $U$, changes relatively slowly with helght in all the cases we examine, we assume that the Scorerparametre $\gamma=N / U$ ( $N$ Is the Vălsäla-Brunt frequency) is the only parametre needed for determining the motion. In the usual way we define layers with constant $\gamma$ and the requirement of no vertical motion at the ground, $x_{3}=0$, and for $x_{3}+\infty$ defines a well-known elgenvalue-problem which in turn leads to the dispersion relations given below.

Subsequently we denote the length of the horizontal wave number vector by $k$ and we introduce an angle $\varphi$ defined by $\cos \varphi=-k_{1} / k$.

1) Discontinuous model: A neutrally stable atmosphere above and below an infinitely thin inversion.

We set $N=0, U=U_{0}$ for $0<x_{3}<H_{0}$ and $N=0, U=U_{1}$ for $x_{3}>H_{0}$ and denote the relative density difference at the Inversion layer by $\varepsilon,(\varepsilon \ll 1)$. The dispersion relation for this model is

$$
D=1+\left(\frac{U_{1}}{U_{0}}\right)^{2} \operatorname{Tan} k H_{0}-\frac{1}{I^{2} \cos ^{2} \varphi} \frac{\operatorname{Tan} k H_{0}}{k H_{0}}=0
$$

where $F=U_{0} / \sqrt{\varepsilon g H_{0}}$ is the Froude number. W1th Tan $k H \simeq \mathbf{k H}$ an approximation to (A 1) vald also for $\mathrm{kH}$ considerably larger than unty is obtained. This approximation is used for evaluating the phase IInes eq. $(3-6)$. 
11) 4 layer model: The thickness of the three lower layers are denoted $H_{1}$ ( $1=0,1,2$; numbered from the ground). The fourth and upper layer extends to infinity. The value of the scorerparametre in the four layers is respectively $\gamma_{i}(i=0,1,2,3)$. We allow for discontinuity in the wind velocity at $\mathrm{x}_{3}=\mathrm{H}_{0}$ and set $U=U_{0}$ for $x_{3}=H_{0}$ and $U=U_{1}$ for $x_{3}=H_{0}+$. With this notation the dispersion relation reads :

$$
\begin{aligned}
D & =q_{2}\left(q_{0} \frac{\operatorname{tg} q_{1} H_{1}}{q_{1}}+\left(\frac{U_{1}}{U_{0}}\right)^{2} \operatorname{Tan} q_{0} H_{0}\right)\left(q_{3}+q_{2} \operatorname{Tan} q_{2} H_{2}\right) \\
& +\left(q_{0}-q_{1}\left(\frac{U_{1}}{U_{0}}\right)^{2} \operatorname{Tan} q_{0} H_{0} \operatorname{tg} q_{1} H_{1}\right)\left(q_{2}+q_{3} \operatorname{Tan} q_{2} H_{2}\right)
\end{aligned}
$$

where

$$
q_{1}=\left[\left(\frac{\gamma_{1}}{\cos \varphi}\right)^{2}-k^{2}\right]^{\frac{1}{2}}
$$

and

$$
q_{1}=\left[x^{2}-\left(\frac{\gamma_{1}}{\cos \varphi}\right)^{2}\right]^{\frac{1}{2}} \quad \text { for } \quad 1=0,2,3
$$

For the trapped wave modes we consider, all the $q_{1}$ are real. 
REFERENCES

1 Blumen, W. and Cary D. McGregor (1976) Tellus 28, 287.

2 Crapper, G.D. (1962) Ph17.Trans Roy. Soc. London Serles A 254, 601 .

3 Fgellheim, K.W. (1973) Teobnical Report No. 16. Horweglan Meteorologieal Institute. (In Norweglan).

4 Kochin (1938)

Trudy Glav. Geofiz. Observ. $\underline{\mathbf{8}}, \mathbf{3}=$ Sobranie Sochịneinı TI p.478 Akad. Nauk. SSSR Moscow-Leningrad 1949 (In Russian).

5 Mohr, T. (1971) Met. Rdsch 24, 30.

6 Palm, E. (1958) Geof. Publ. (Oslo) Vol. XX, No. 3.

7 Scorer, R.S. and Mary Wilkinson (1956) Quart.J.R.Met.Soc. 82, 419 .

8 Wehper, I. (1949) Dissertation Universität Leipzig Akademie-Verlag, Berlin.

9 Whitham, G.B. (1974) Iinear and Nonlinear Waves, John Wiley \& Sons, New York.

10 Wurtele, M.G. (1957) Be1tr. Phys. Atmos. 29, 242. 


\section{FIGURE CAPTIONS}

Figure 1

Maps of Jan Mayen, The Bear Island and Hopen showing the main topographical features of the island $\mathbf{s}$.

F1gure 2

Section of a VHRR-photograph taken by NOAA 5 . The scale indicates the flight direction of the satelilte. The scale perpendicular to the flight direction is subpressed by a factor: 0.72

2a; Jan Mayen Sept. 1. 1976, 11.17 GMT (visual band) On the left side Scoresby Sound on EastGreenland.

2b; Jan Mayen Dec. 29. 1976, 11.22 GMT (Infrared band).

2c; Jan Mayen Oct. 8. 1976, 13.14 GMT (infrared band).

2d; Spitzbergen Sept. 19.1976, 11.37 GMT (visual band).

Figure 3 Upper air data on temperature (solid Iines), dew-point temperature (dotted lines), wind (direction and force, in kns.) and stability (N). In the temperature diagram (left) wet adlabats are drawn by -o- and dry adiabats by - - .

3a; Jan Mayen Sept. 1. 1976, 11.15 GMT - 12.20 GMT. 3b; Jan Mayen Dec. 29. 1976, 11.15 GMT - 11.53 GMT. 3c; Jan Mayen Oct. 8. 1976, 11.15 GMT - 12.27 GMT. 3d; The Bear Island Sept. 19.1976, 11.15 GMT 12.34 GMT. 
Figure $4 a$

The semi wedge-angle, $\theta$, as function of $\gamma_{I}$ for models with $U / U_{0}=0.8, H_{0}=1700 \mathrm{~m}, \mathrm{H}_{2}=900 \mathrm{~m}$ and $\mathrm{H}_{2}=6700 \mathrm{~m} \cdot \mathrm{A}: \gamma_{0}=\gamma_{2}=\gamma_{3}=0$.

$B: \gamma_{0}=0, \gamma_{2}=0.2 \cdot 10^{-3} \mathrm{~m}^{-1}, \gamma_{3}=0.3 \cdot 10^{-3} \mathrm{~m}^{-1}$. $c: r_{0}=0, \gamma_{2}=0.4 \cdot 10^{-3} \mathrm{~m}^{-1}$ and $r_{3}=0.5 \cdot 10^{-3} \mathrm{~m}^{-1}$. Solld lines and dotted lines correspond respectively to the 1. mode and the 2. mode. Case III is marked with a dot.

Figure 4b The semi wedge-angle, $\theta$, as function of $r_{1}$ for models with $U / U_{0}=1.0$ for models with $H_{0}=700 \mathrm{~m}$, $\mathrm{H}_{1}=800 \mathrm{~m}, \mathrm{H}_{2}=1700 . \mathrm{A}: r_{0}=r_{2}=r_{3}=0$. B: $\quad \gamma_{0}=0, \quad \gamma_{2}=0.2 \cdot 10^{-3} \mathrm{~m}^{-1}, \quad \gamma_{3}=0.3 \cdot 10^{-3} \mathrm{~m}^{-1}$. C: $r_{0}=0, r_{2}=0.3 \cdot 10^{-3} \mathrm{~m}^{-1}$ and $r_{3}=0.55 \cdot 10^{-3} \mathrm{~m}^{-1}$. Solld I1nes correspond respectively to the 1. mode and the 2. mode. Case IV is marked with a dot.

F1gure 5 Phase-Iines for Case I-IV displayed respectively 1. $b$. The phase difference between corresponding points on neighbouring lines are $2 \pi$. 


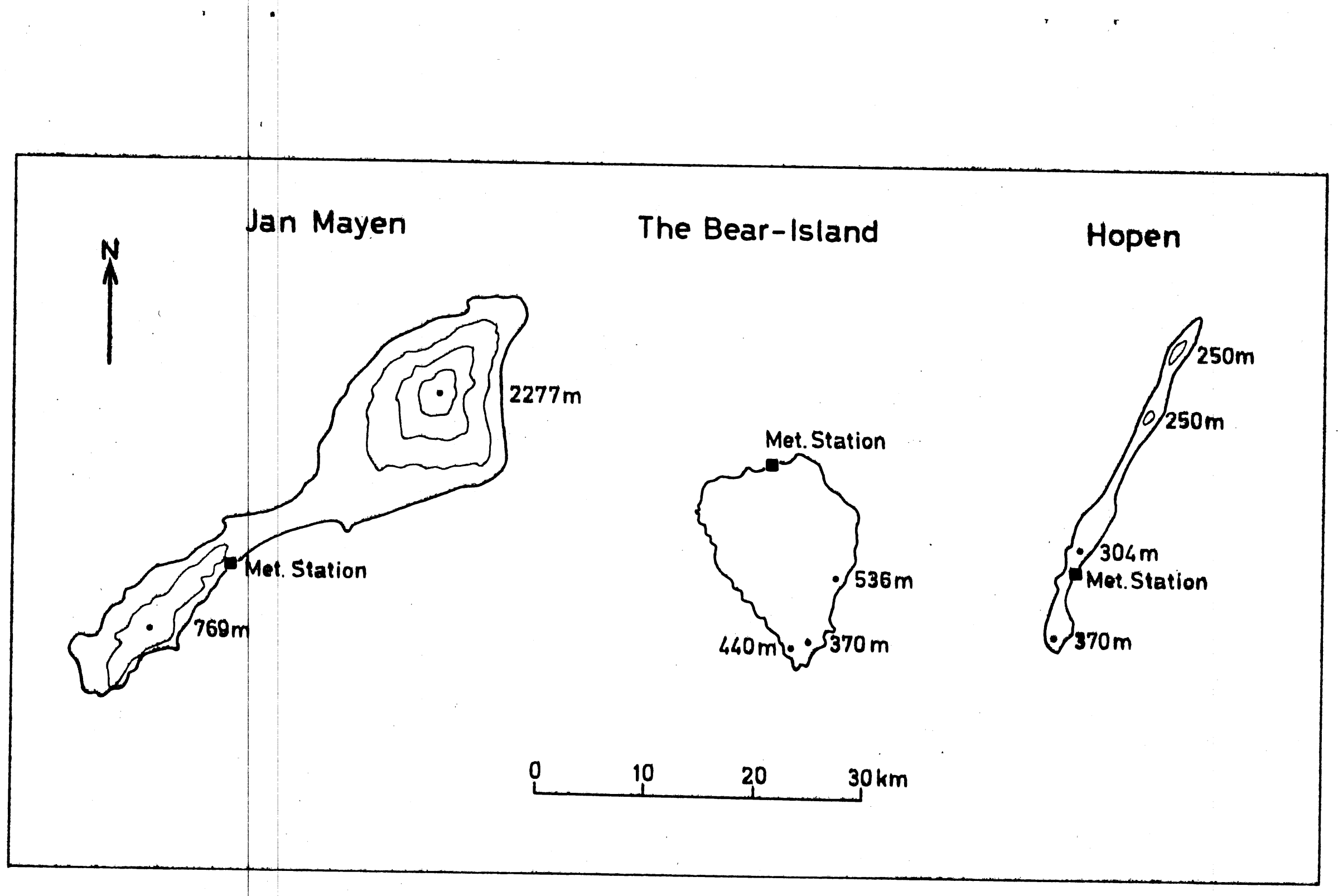

Fig. 1 

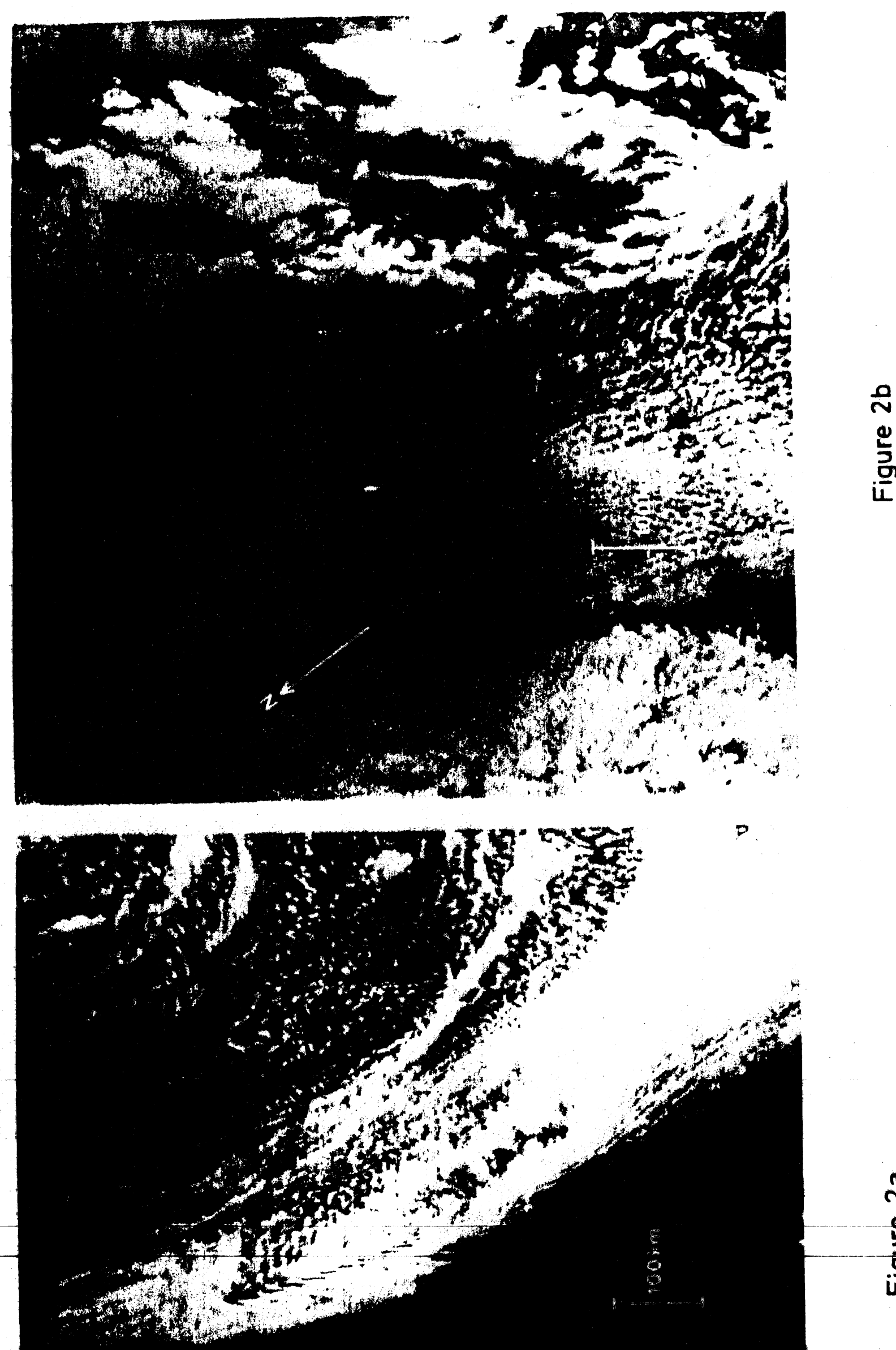


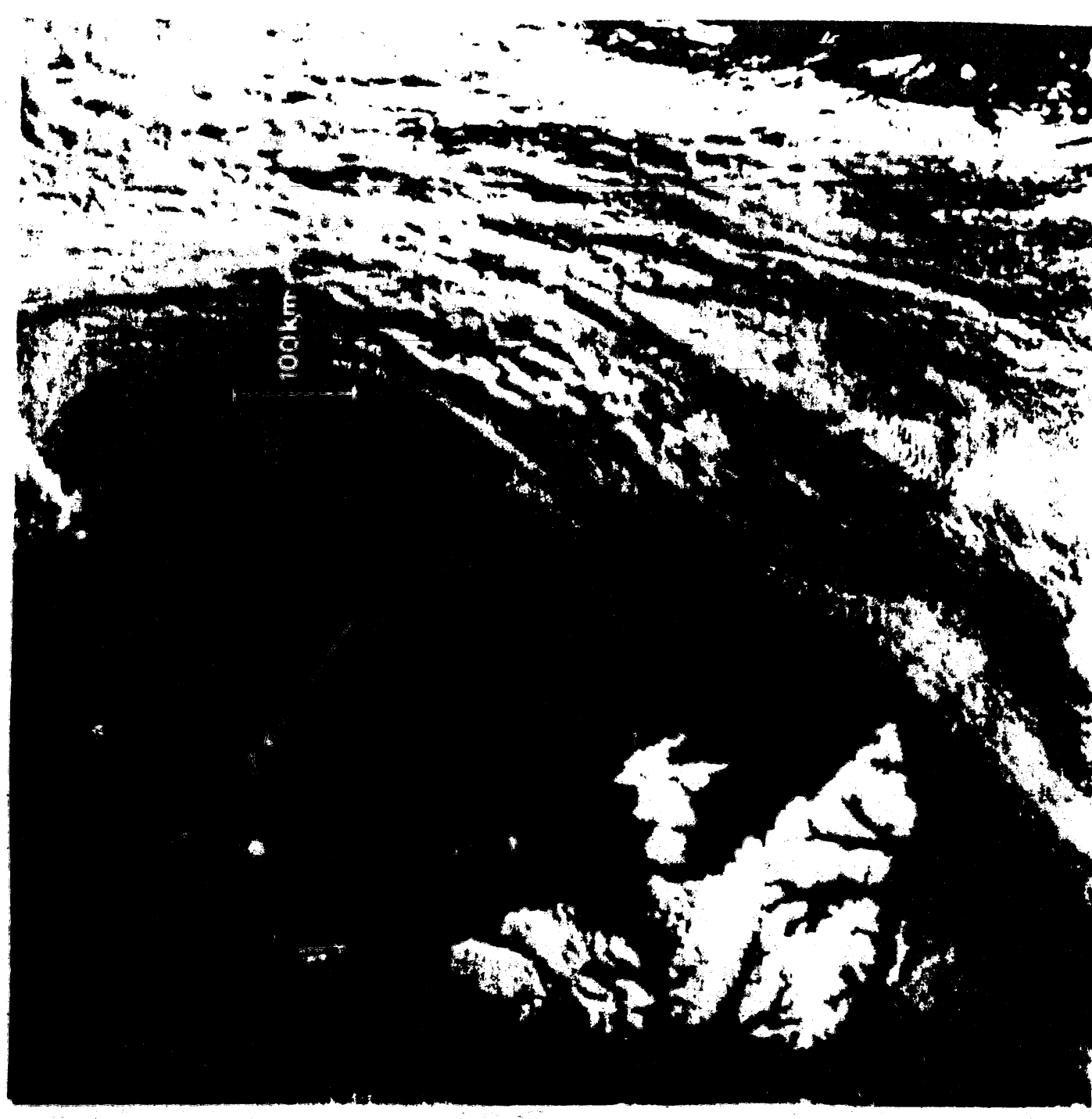

몬

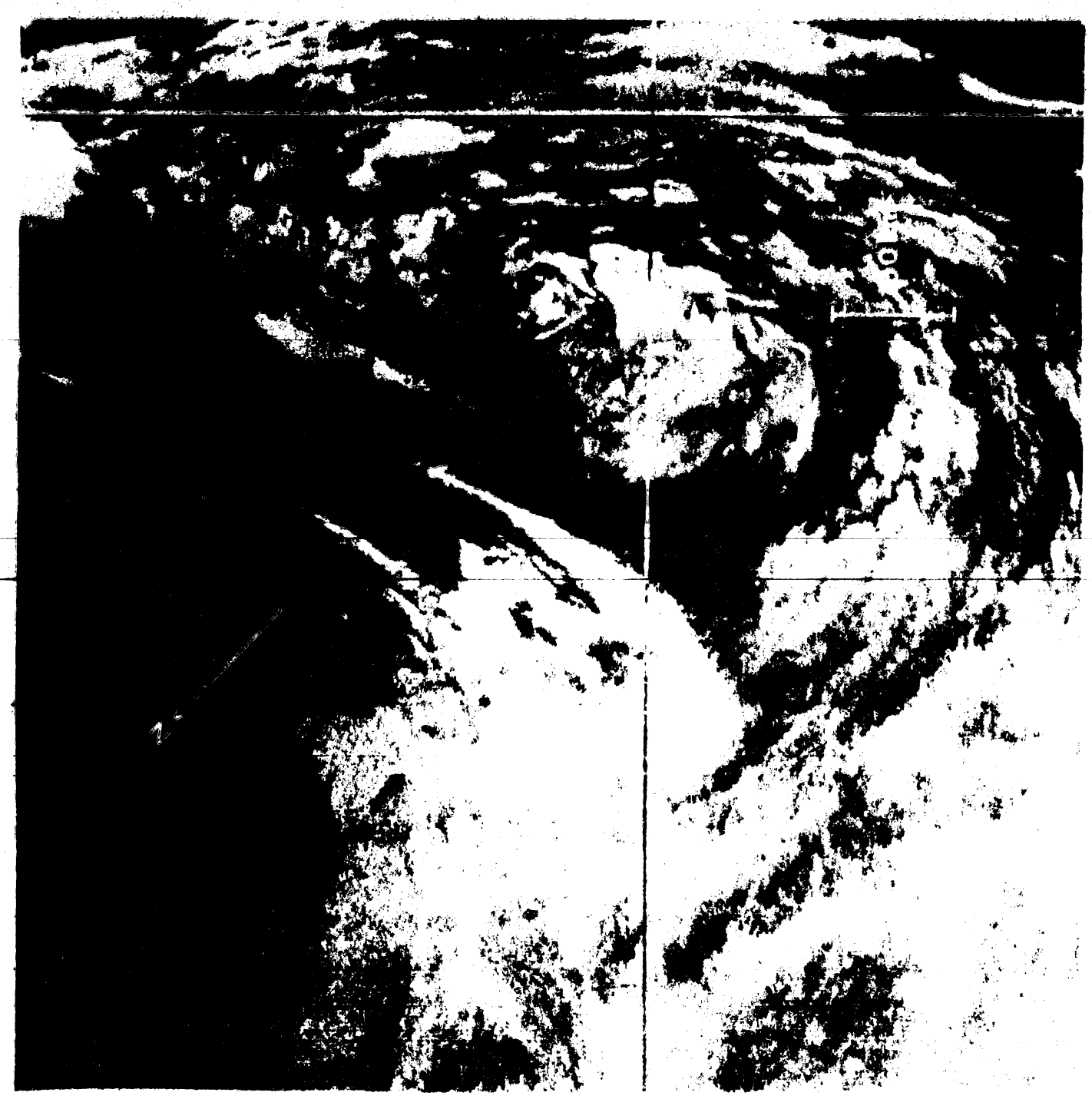

盛 


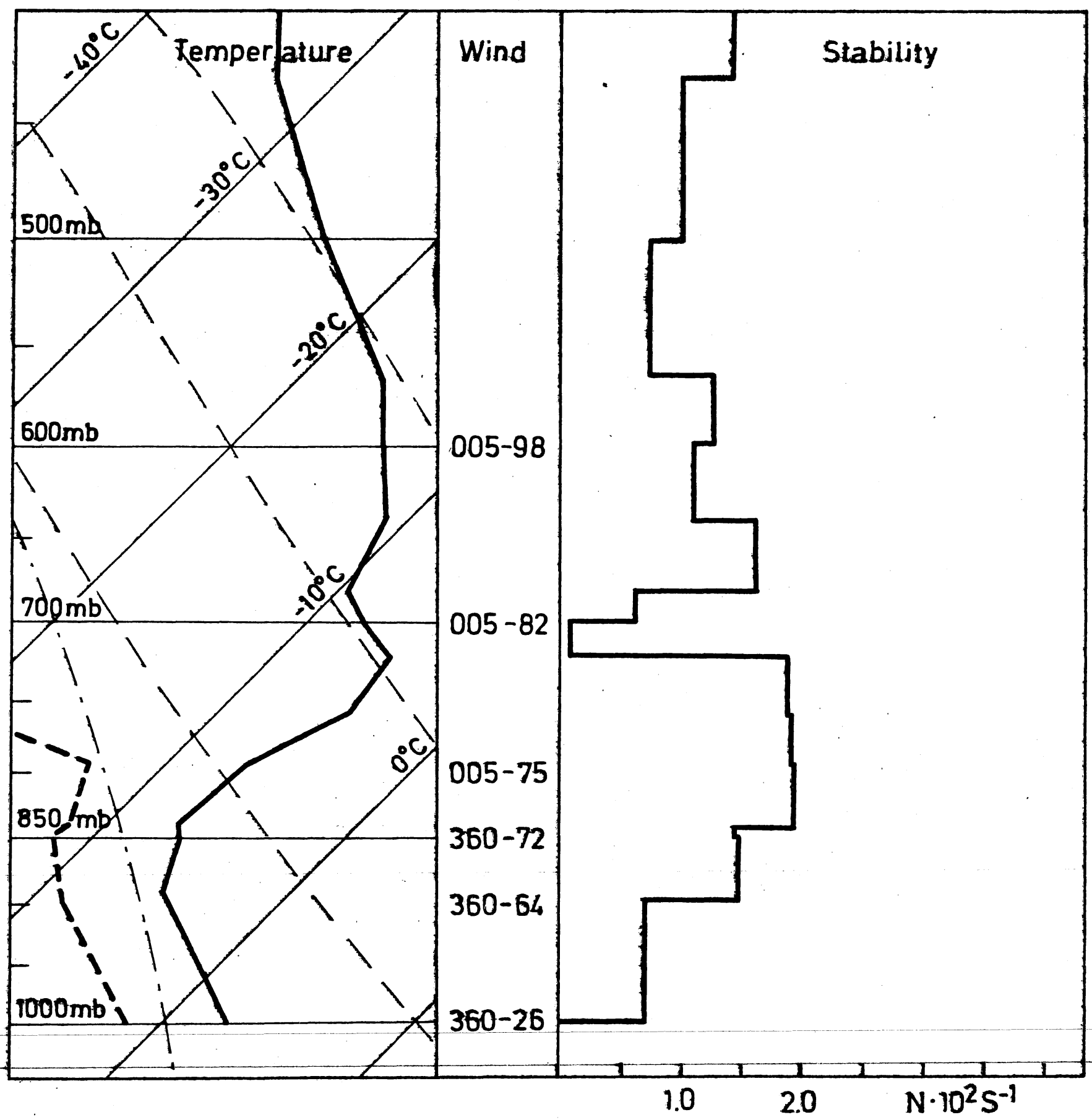

Fig. 3a 


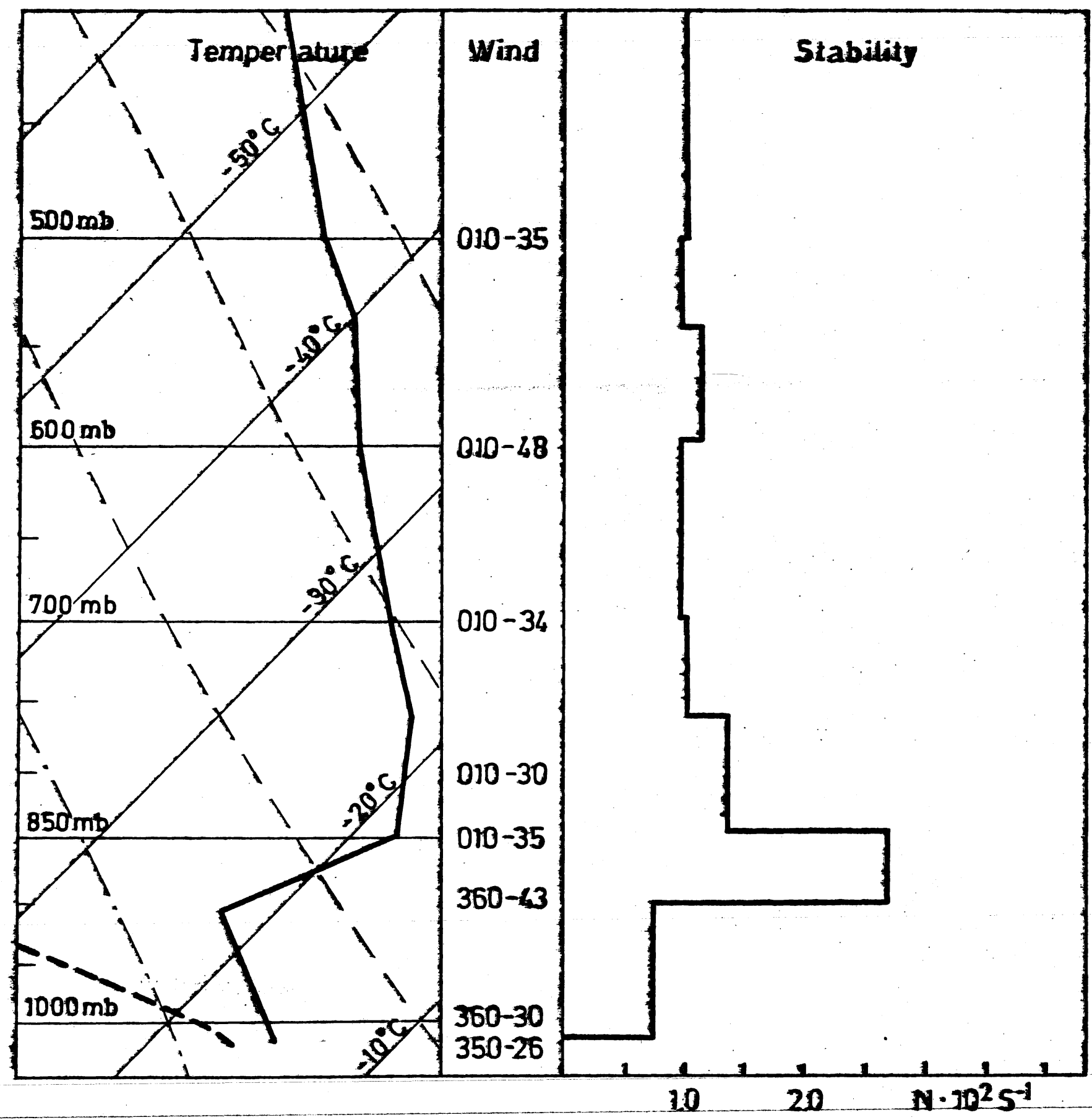

Fig. 36 


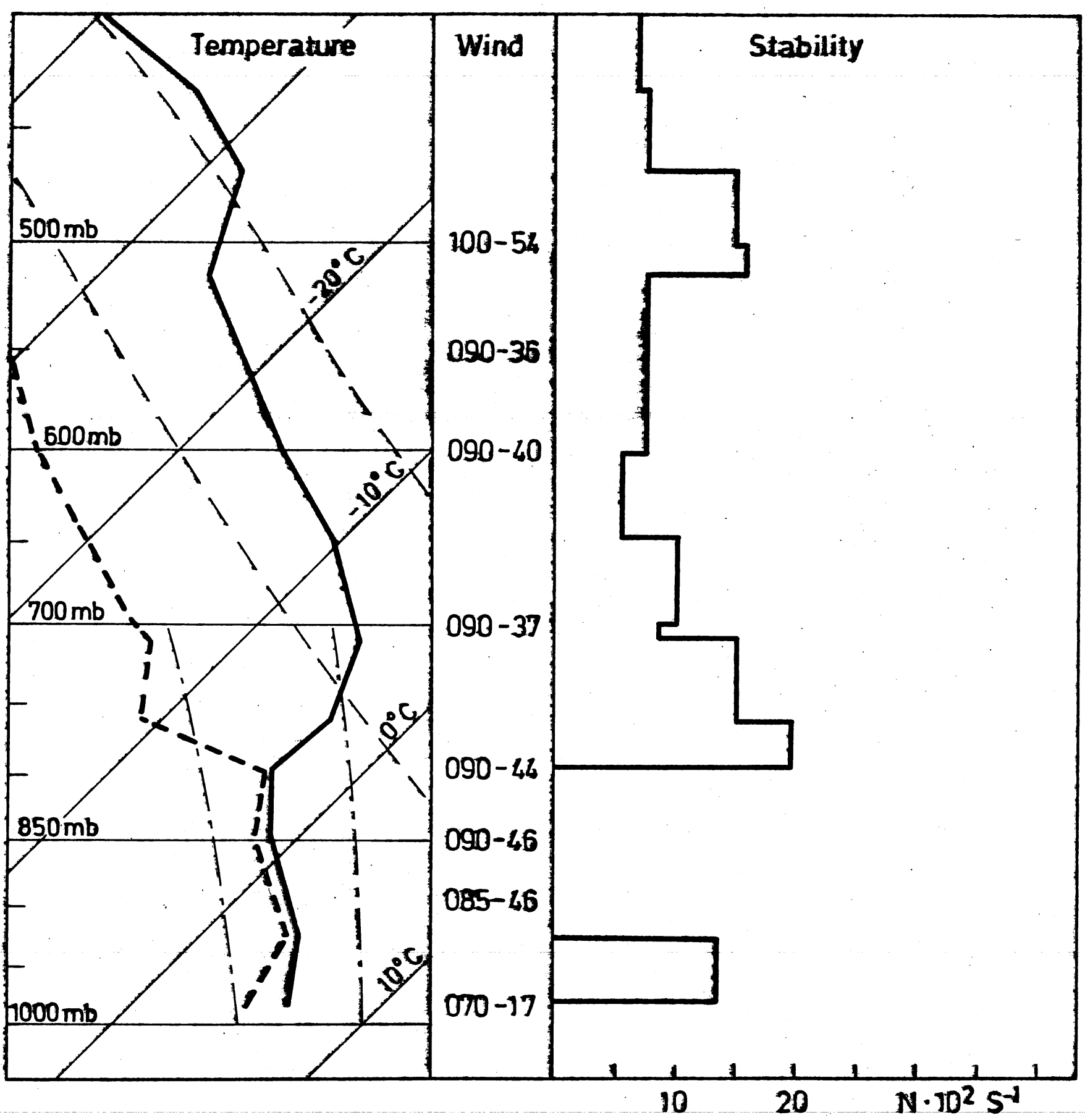

Fig. $3 c$ 


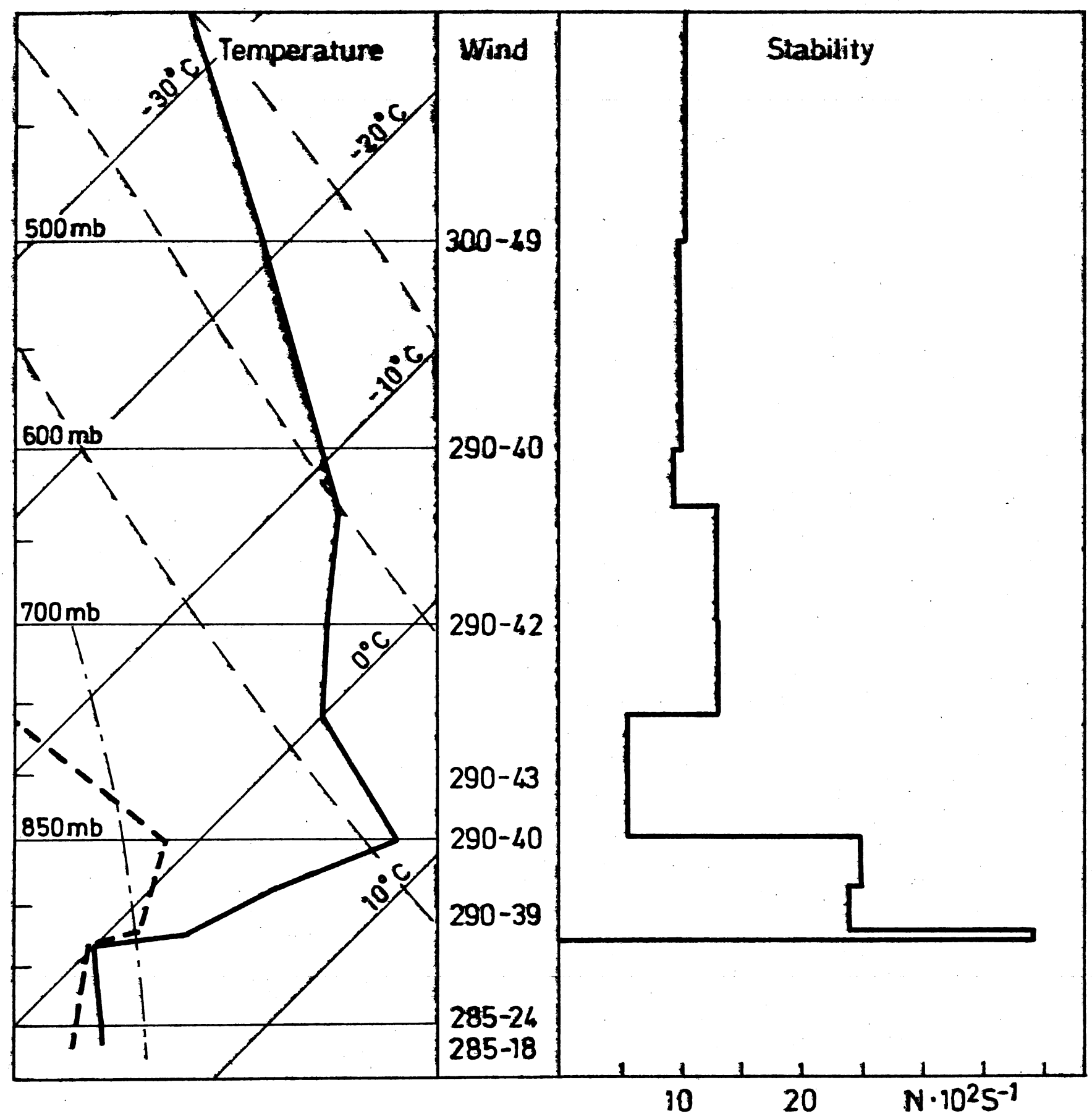

Fig. 3d 


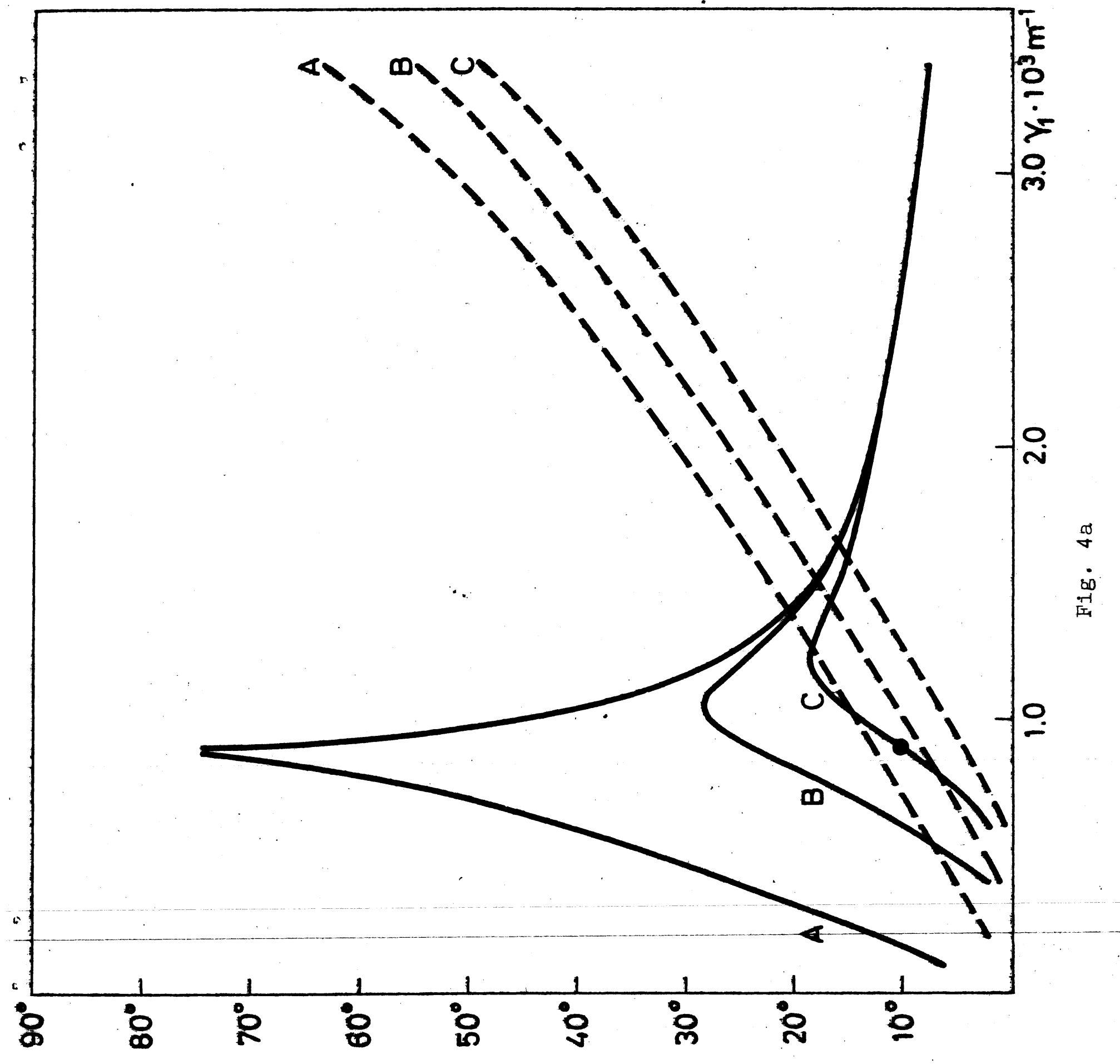




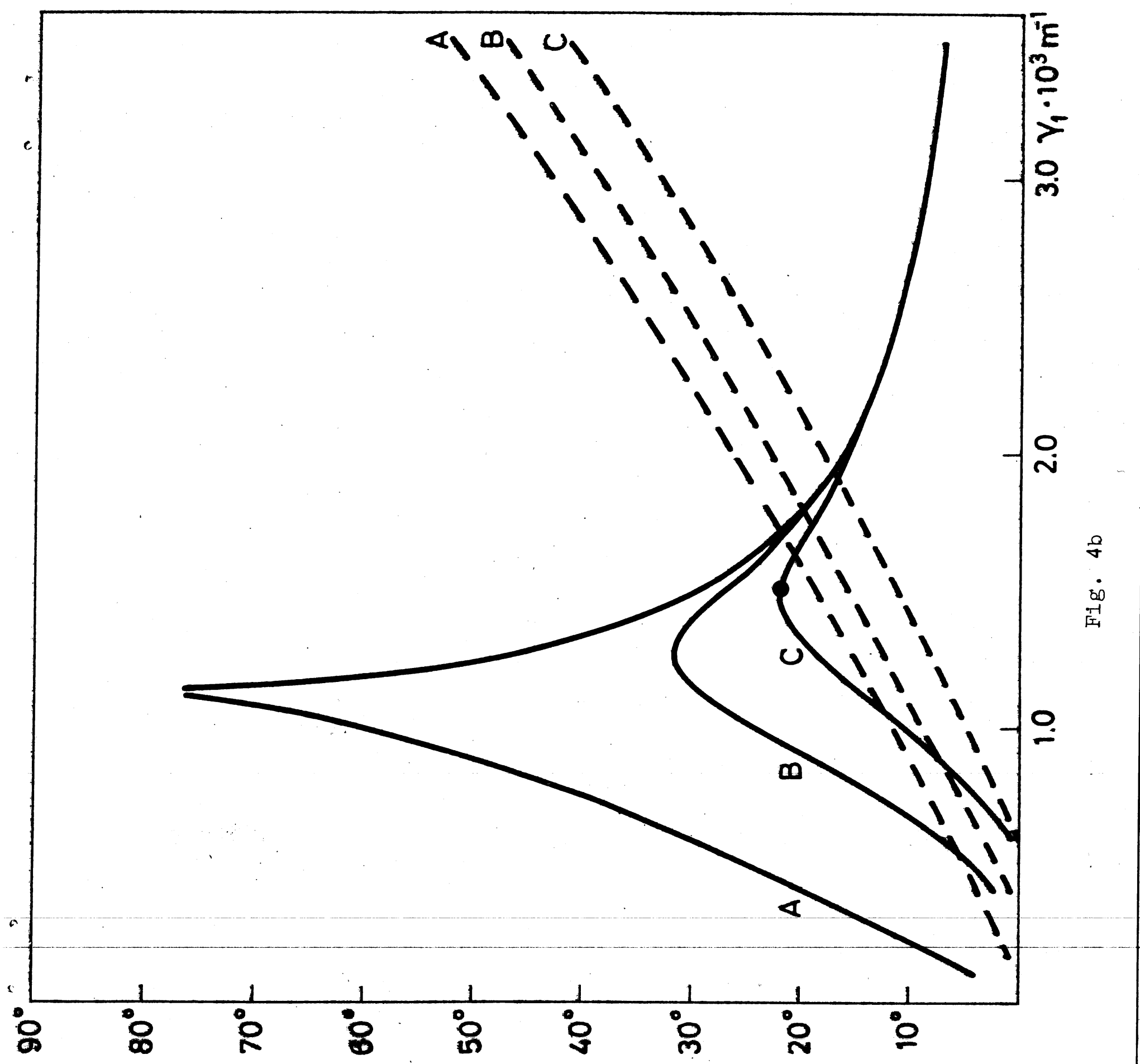




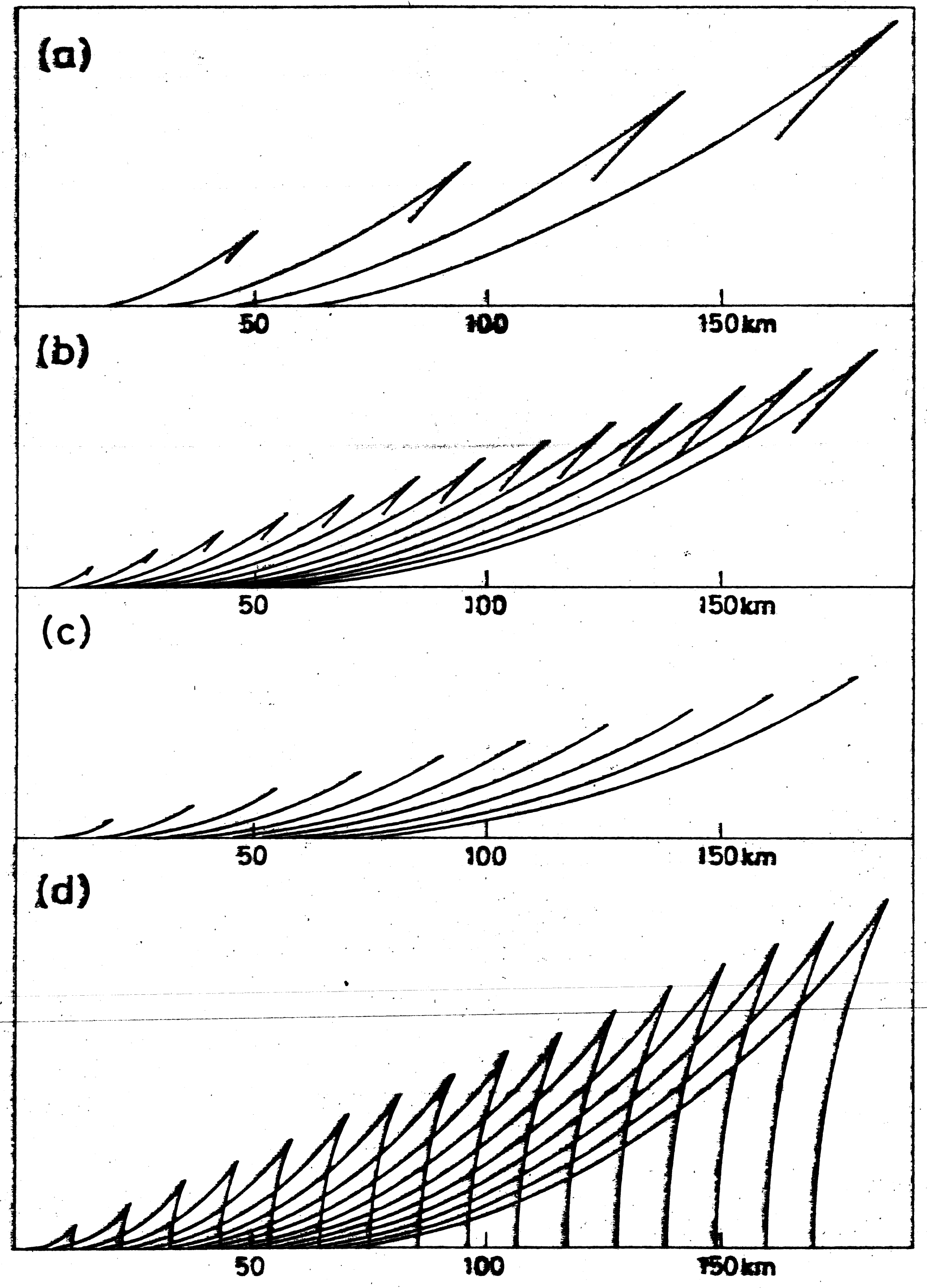

Fig. 5 